\title{
THE DENJOY-WOLFF THEOREM FOR CONDENSING MAPPINGS IN A BOUNDED AND STRICTLY CONVEX DOMAIN IN A COMPLEX BANACH SPACE
}

\author{
Monika Budzyńska \\ Uniwersytet Marii Curie-Skłodowskiej, Instytut Matematyki \\ 20-031 Lublin, Poland; monikab1@hektor.umcs.lublin.pl
}

\begin{abstract}
If $D$ is a bounded and strictly convex domain in a complex Banach space and $f: D \rightarrow D$ is holomorphic, condensing with respect to the Kuratowski measure of noncompactness and fixed-point-free, then there exists $\xi \in \partial D$ such that the sequence $\left\{f^{n}\right\}$ of the iterates of $f$ converges in the compact-open topology to the constant mapping taking the value $\xi$.
\end{abstract}

\section{Introduction}

The present paper is devoted the Denjoy-Wolff theorem in bounded and strictly convex domains in complex Banach spaces. Recall that in 1926 Denjoy and Wolff $[23,71,72]$, see also $[73,18]$, proved the following theorem.

Theorem 1.1. Let $\Delta$ be the open unit disc in the complex plane $\mathbf{C}$. If an analytic function $f: \Delta \rightarrow \Delta$ does not have a fixed point, then there is a unique point $\xi$ in $\partial \Delta$ such that the iterates $f^{n}$ of $f$ converge to $\xi$, uniformly on compact subsets of $\Delta$.

Comparing the above Denjoy-Wolff theorem with the Riemann theorem and the Osgood, Taylor and Carathéodory theorem [17] we get the following version of the Denjoy-Wolff theorem for fixed-point-free self-mappings of bounded and convex domains in the complex plane $\mathbf{C}$.

Theorem 1.2. Let $D$ be a bounded and convex domain in $\mathbf{C}$. If an analytic function $f: D \rightarrow D$ is fixed-point-free, then there exists $\xi \in \partial D$ such that the sequence $\left\{f^{n}\right\}$ of the iterates of $f$ converges in the compact-open topology to the constant mapping taking the value $\xi$.

The last theorem is not valid in $\mathbf{C}^{n}$ for $n \geq 2$. But there are positive results of this type. The most general one is due to the author, who used the horosphere method, which was introduced by Abate [1,2], to prove the following theorem for bounded and strongly convex domains in $\mathbf{C}^{n}$ (for earlier results in $\mathbf{C}^{n}$, see $[1,2,21$, $35,45,47,56,68])$.

Theorem 1.3. ([12], see also [3]) If $D$ is a bounded and strictly convex domain in $\mathbf{C}^{n}$ and $f: D \rightarrow D$ is holomorphic and fixed-point-free, then there exists $\xi \in \partial D$ such that the sequence $\left\{f^{n}\right\}$ of iterates of $f$ converges in the compact-open topology to the constant mapping taking the value $\xi$.

Recall here that a bounded and convex domain $D$ in a complex Banach space $(X,\|\cdot\|)$ is strictly convex if for each $x, y \in \bar{D}$, the open segment

doi:10.5186/aasfm.2014.3944

2010 Mathematics Subject Classification: Primary 32A10, 32A17, 46G20, 47H10.

Key words: Horosphere, iterates of holomorphic mappings, the Denjoy-Wolff theorem, the Kobayashi distance. 


$$
(x, y)=\{z \in X: z=s x+(1-s) y \text { for some } 0<s<1\}
$$

lies in $D([31])$

But in a complex infinite dimensional Banach space the Denjoy-Wolff theorem in the above form is false. Namely, Stachura shows that in the complex infinite dimensional Hilbert space $H=l^{2}$ the convergence result fails even for biholomorphic self-maps in the open unit ball $B_{H}$ ([69], see also [28]). So, in order to obtain a generalization of the Denjoy-Wolff theorem we have to impose some restrictions on the holomorphic self-mapping $f: D \rightarrow D$. Then, using the methods similar to those which were applied in [12], the following Denjoy-Wolff theorem for compact mappings was proved in [16] (in case of the open and strictly convex unit ball $B$ in a complex Banach space $(X,\|\cdot\|)$, see $[22,37,44,65])$.

Theorem 1.4. ([16], see also [15]) If $D$ is a bounded and strictly convex domain in a complex Banach space $(X,\|\cdot\|)$, and $f: D \mapsto D$ is compact, holomorphic and fixed-point-free, then there exists a point $\xi \in \partial D$ such that the sequence $\left\{f^{n}\right\}$ of the iterates of $f$ converges in the bounded-open topology to the constant map taking the value $\xi$, i.e., on each $k_{D}$-bounded subset $C$ of $D$, the sequence $\left\{f^{n}\right\}$ tends uniformly to $\xi$.

The main result of our paper is the Denjoy-Wolff theorem for condensing $k_{D^{-}}$ nonexpansive mapping ( $k_{D}$ denotes the Kobayashi distance in $\left.D\right)$ in the bounded and strictly convex domain $D$ (in case of the open and strictly convex unit ball $B$ in $(X,\|\cdot\|)$, see $[38,48]$ and Remark 3.1 in [15]). Note that the class of all condensing mappings contains the class of all compact mappings. Observe also that in the infinitely dimensional complex Banach space $(X,\|\cdot\|)$ the assumption, that the $k_{D}$-nonexpansive mapping $f: D \rightarrow D$ is condensing, is very natural for our purposes since this assumption guarantees us that for each $x \in D$ the set of all elements of the sequence $\left\{f^{n}(x)\right\}$ is relatively compact in $(X,\|\cdot\|)([67])$.

Unfortunately, in case of condensing and holomorphic mappings we are not able to use the horosphere method since it is not known whether all horospheres are nonempty. Therefore in this paper we present a new approach in the proof of the Denjoy-Wolff theorem for condensing and holomorphic mappings $f: D \rightarrow D$. To get a contradiction we use a method of a minimal $f$-invariant set $C \subset D$ and a new intrinsic metric $k_{D, C}$ in $C$.

The paper is organized in the following way. In Section 2 we provide the necessary prerequisities for our results. These concern the Kobayashi distance $k_{D}, k_{D^{-}}$ nonexpansive and condensing mappings, and we also introduce an intrinsic metric $k_{D, C}$ in a $k_{D}$-closed and convex subset $C$ of a domain $D$. But for the better reading we give, in the last section of our paper, the proofs of the properties of this intrinsic metric $k_{D, C}$, since these proofs have a very technical character. In the section 3 we state and prove our main theorem and in the next section we present the DenjoyWolff theorem for semigroups. As we mentioned earlier in the Section 5 we investigate an intrinsic metric $k_{D, C}$ in a $k_{D}$-closed and convex subset $C$ of a domain $D$ and we establish Theorem 5.9 which is crucial in the proof of our main theorem.

Finally, note that there are many papers on the Denjoy-Wolff theorem, the Denjoy-Wolff type theorem and their applications, see e.g. [2, 8, 9, 13, 14, 32, 33, 39, $46,53,54,55,57,60,61,62,63,64,65,66,70]$. 


\section{The Kobayashi distance and its properties}

Since in the proofs of the Denjoy-Wolff theorem the Kobayashi distance ([41] and for more information, see [2, 24, 25, 26, 30, 33, 34, 36, 42, 43, 46, 52]) plays the main role we recall a few important properties of the Kobayashi distance, which will be used in the proof of our main theorem.

We begin with the definition of the Poincaré distance. Let $\Delta$ be the open unit disc in the complex plane $\mathbf{C}$. Recall that the Poincaré distance $\rho_{\Delta}$ on $\Delta$ is given by

$$
k_{\Delta}(z, w)=\rho_{\Delta}(z, w):=\arg \tanh \left|\frac{z-w}{1-z \bar{w}}\right|=\arg \tanh (1-\sigma(z, w))^{\frac{1}{2}},
$$

where

$$
\sigma(z, w):=\frac{\left(1-|z|^{2}\right)\left(1-|w|^{2}\right)}{|1-z \bar{w}|^{2}}, \quad z, w \in \Delta .
$$

Now let $D$ be a bounded and convex domain in a complex Banach space $(X,\|\cdot\|)$. We use the following definition of the Kobayashi distance on $D$. This definition is, in fact, the definition of the Lempert function $\delta[52,26]$, i.e.,

$$
\begin{gathered}
k_{D}(x, y)=\delta_{D}(x, y):=\inf \left\{\rho_{\Delta}(0, \lambda): \lambda \in[0,1) \text { and there exists } f \in H(\Delta, D)\right. \\
\text { so that } f(0)=x, f(\lambda)=y\},
\end{gathered}
$$

where $H(\Delta, D)$ denotes the family of all holomorphic mappings $f: \Delta \rightarrow D$.

Here, we present a few basic properties of the Kobayashi distance.

Lemma 2.1. [34] Let $(X,\|\cdot\|)$ be a complex Banach space, $D \subset X$ be a bounded and convex domain and let $k_{D}$ be the Kobayashi distance. The Kobayashi distance $k_{D}$ is locally equivalent to the norm $\|\cdot\|$ in $X$, i.e., the following inequalities are valid:

(i)

$$
\arg \tanh \left(\frac{\|x-y\|}{\operatorname{diam} D}\right) \leq k_{D}(x, y)
$$

(ii)

for all $x, y \in D$, where $\operatorname{diam} D:=\sup \{\|w-z\|: w, z \in D\}$;

$$
k_{D}(x, y) \leq \arg \tanh \left(\frac{\|x-y\|}{\operatorname{dist}(x, \partial D)}\right)
$$

whenever $\|x-y\|<\operatorname{dist}(x, \partial D)$, where $\operatorname{dist}(x, \partial D):=\inf \{\|x-y\|: y \in \partial D\}$.

Lemma 2.2. [51, 38, 48] Let $D$ be a convex and bounded domain in a complex Banach space $(X,\|\cdot\|)$.

(i) If $x, y, w, z \in D$ and $s \in[0,1]$, then

$$
k_{D}(s x+(1-s) y, s w+(1-s) z) \leq \max \left[k_{D}(x, w), k_{D}(y, z)\right] ;
$$

(ii) if $x, y \in D$ and $s, t \in[0,1]$, then

$$
k_{D}(s x+(1-s) y, t x+(1-t) y) \leq k_{D}(x, y) .
$$

Next, recall that a nonempty subset $C$ of $D$ lies strictly inside $D$ if

$$
\operatorname{dist}(C, \partial D):=\inf \{\|x-y\|: x \in C, y \in \partial D\}>0 .
$$

Observe that a nonempty subset $C$ of a bounded and convex domain $D$ is $k_{D^{-}}$ bounded if and only if $C$ lies strictly inside $D([34])$.

The next property of $k_{D}$ is the following. 
Lemma 2.3. $[15,38,46]$ Let $D$ be a bounded and strictly convex domain in a complex Banach space $(X,\|\cdot\|)$. Let $\left\{x_{j}\right\}_{j \in J}$ and $\left\{y_{j}\right\}_{j \in J}$ be two nets in $D$ which converge in norm to $\xi \in \partial D$ and to $\eta \in \bar{D}$, respectively. If

$$
\sup \left\{k_{D}\left(x_{j}, y_{j}\right): j \in J\right\}=c<\infty,
$$

then $\xi=\eta$.

Now we recall a few results showing basic connections between holomorphic mappings and the Kobayashi distance. Let $D_{1}$ and $D_{2}$ be bounded convex domains in complex Banach spaces $\left(X_{1},\|\cdot\|_{1}\right)$ and $\left(X_{2},\|\cdot\|_{2}\right)$, respectively. A mapping $f: D_{1} \rightarrow D_{2}$ is nonexpansive with respect to the Kobayashi distance if

$$
k_{D_{2}}(f(x), f(y)) \leq k_{D_{1}}(x, y)
$$

for all $x, y \in D$. Note that each holomorphic mapping $f: D_{1} \rightarrow D_{2}$ is nonexpansive with respect to the Kobayashi distance. Next, if $D_{1}=D_{2}=D$, then we say that $f$ is $k_{D}$-nonexpansive. So, if $D$ is a bounded domain in a complex Banach space $(X,\|\cdot\|)$, then each holomorphic $f: D \mapsto D$ is $k_{D}$-nonexpansive $[2,24,30,33,34,36,41,66]$.

Let $D$ be a bounded domain in a complex Banach space $(X,\|\cdot\|)$. A mapping $f: D \rightarrow D$ is said to map $D$ strictly inside $D$ if $f(D)$ lies strictly inside $D$. Such a mapping is a $k_{D^{-}}$contraction according to the following Earle-Hamilton theorem.

Theorem 2.4. [27] Let $D$ be a bounded domain in a complex Banach space $(X,\|\cdot\|)$. If a holomorphic $f: D \rightarrow D$ maps $D$ strictly inside itself, then there exists $0 \leq t<1$ such that

$$
k_{D}(f(x), f(y)) \leq t k_{D}(x, y)
$$

for all $x$ and $y$ in $D$.

Hence, if $D$ is a bounded and convex domain in a Banach space $(X,\|\cdot\|)$, then by the Earle-Hamilton theorem the mapping $g_{s, z}:=(1-s) z+s \cdot I: D \rightarrow D$ is a $k_{D}$-contraction for each $z \in D$ and $0 \leq s<1$ (here $I$ denotes the identity mapping). Thus for each $k_{D}$-nonexpansive mapping $f: D \rightarrow D$ the mapping $f_{s, z}:=g_{s, z} \circ f=$ $(1-s) z+s f: D \rightarrow D$ is a $k_{D^{-c o n t r a c t i o n}}$ and has exactly one fixed point which we denote by $h_{f}(s, z)$. Fix $0 \leq s<1$ and $x_{0} \in D$. Then the mapping $h_{f}(s, \cdot): D \rightarrow D$ is $k_{D}$-nonexpansive (holomorphic if $f$ is holomorphic) as a limit of the sequence $\left\{f_{s, \cdot}^{n}\left(x_{0}\right)\right\}$.

We will also use the notions of total boundedness, finite total boundedness and finite compactness of a metric space. Recall that a metric space $(Y, d)$ is said to be totally bounded if for each $\varepsilon>0$, it can be decomposed into a finite number of sets of diameter $<\varepsilon([49]$, see also $[29,50])$. We also say that a metric space $(Y, d)$ is finitely totally bounded (finitely compact) if each nonempty and bounded subset of $Y$ (each nonempty, bounded and closed subset of $Y$ ) is totally bounded (compact). Now we are able to recall Całka's theorem regarding the behavior of the sequence of iterates of a nonexpansive mapping on a finitely totally bounded metric space $(Y, d)$.

Theorem 2.5. [20] Let $f$ be a nonexpansive mapping of a finitely totally bounded metric space $(Y, d)$ into itself. If for some $y_{0} \in Y$, the sequence $\left\{f^{n}\left(y_{0}\right)\right\}$ contains a bounded subsequence, then for each $y \in Y$, the sequence $\left\{f^{n}(y)\right\}$ is bounded.

Now we recall the definition of a condensing mapping. 
Definition 2.1. $[4,6,7,31]$ Let $(Y, d)$ be a metric space and let $\emptyset \neq D \subset Y$. We say that a mapping $f: D \rightarrow D$ is $\alpha_{d}$-condensing with respect to Kuratowski's measure of noncompactness $\alpha_{d}$ ([49]) if

$$
\alpha_{d}(f(C))<\alpha_{d}(C)
$$

for each bounded $C \subset D$ with $\alpha_{d}(C)>0$.

Observe that condensing mappings have the following properties.

Lemma 2.6. Let $D$ be a convex bounded domain in a Banach space $(X,\|\cdot\|)$ and let $f: D \rightarrow D$ be $k_{D}$-nonexpansive and condensing with respect to $\alpha_{\|\cdot\|}$.

(i) [67] For each $x \in D$ we have

$$
\alpha_{\|\cdot\|}\left(\left\{f^{n}(x): n=1,2, \ldots\right\}\right)=0
$$

and therefore the set of iterates $\left\{f^{n}(x): n=1,2, \ldots\right\}$ of $x$ is finitely totally bounded in $\left(D, k_{D}\right)$ and the closure in $(X,\|\cdot\|)$ of this set is compact in $(X,\|\cdot\|)$.

(ii) [38] If $C$ is a nonempty, $k_{D}$-closed and f-invariant subset of $D,\left\{s_{n}\right\}$ is a sequence such that $\lim _{n \rightarrow \infty} s_{n}=1,0<s_{n}<1$ and $\left\{z_{n}\right\}$ is a sequence of elements of $C$, then the sequence $\left\{x_{n}\right\}$, given by $x_{n}=h_{f}\left(s_{n}, z_{n}\right)=f_{s_{n}, z_{n}}\left(x_{n}\right)=$ $\left(1-s_{n}\right) z_{n}+s_{n} f\left(x_{n}\right)$ for each $n \in \mathbf{N}$, contains a norm-convergent subsequence.

Directly from the above lemma, the Earle-Hamilton theorem and the Całka theorem we get the following theorem which is basic for our next considerations.

Theorem 2.7. [38] Let $D$ be a convex bounded domain in a complex Banach space $(X,\|\cdot\|)$ and let $k_{D}$ denote its Kobayashi distance. If $f: D \rightarrow D$ is $k_{D^{-}}$ nonexpansive and condensing with respect to $\alpha_{\|\cdot\|}$, then the following conditions are equivalent:

(i) $f$ has a fixed point;

(ii) there exist $x \in D$ and a $k_{D}$-bounded subsequence of its iterates $\left\{f^{n_{i}}(x)\right\}$;

(iii) there exists $x \in D$ with a $k_{D}$-bounded sequence of its iterates $\left\{f^{n}(x)\right\}$;

(iv) for each $x \in D$ the sequence of its iterates $\left\{f^{n}(x)\right\}$ is $k_{D}$-bounded;

(v) there exists a nonempty, $k_{D}$-closed, convex, $k_{D}$-bounded and $f$-invariant subset $C$ of $D$;

(vi) there exists a nonempty, $k_{D}$-bounded and $f$-invariant subset $C$ of $D$;

(vii) there exists a $k_{D}$-bounded and norm convergent sequence $\left\{x_{n}\right\}$ such that $\left(f\left(x_{n}\right)-x_{n}\right) \rightarrow 0$

(viii) there exists a $k_{D}$-bounded sequence $\left\{x_{n}\right\}$ such that $\left(f\left(x_{n}\right)-x_{n}\right) \rightarrow 0$.

Using Theorem 2.7, Lemma 2.3 and Lemma 2.6 we obtain two important theorems about $k_{D}$-nonexpansive, fixed-point-free and condensing mappings. To make the paper self-contained we give them with their proofs.

Theorem 2.8. Let $D$ be a strictly convex bounded domain in a complex Banach space $(X,\|\cdot\|)$ and let $k_{D}$ denote its Kobayashi distance. If $f: D \rightarrow D$ is $k_{D}$-nonexpansive, fixed-point-free and condensing with respect to $\alpha_{\|\cdot\|}$, then for each $x \in D$ the set $A$ of all accumulation points of the sequence $\left\{f^{n}(x)\right\}$ has the following properties:

(i) $A \neq \emptyset$,

(ii) $A \subset \partial D$, 
(iii) the set $A$ is independent of the choice $x \in D$.

Proof. Since $f$ is fixed-point-free, Theorem 2.7 (ii) implies that for each $x \in D$ we have

$$
\lim _{n \rightarrow \infty} k_{D}\left(f^{n}(x), x\right)=\infty .
$$

By Lemma 2.6 (i) the closure in $(X,\|\cdot\|)$ of the set of iterates $\left\{f^{n}(x)\right\}$ is compact in $(X,\|\cdot\|)$. Let $A$ denote the set of all accumulation points of the sequence $\left\{f^{n}(x)\right\}$. Then we have $\emptyset \neq A \subset \partial D$. Observe that

$$
k_{D}\left(f^{n}(x), f^{n}(x)\right) \leq k_{D}(x, y)
$$

for $n=1,2, \ldots$ and each $x, y \in D$ and therefore, by Lemma 2.3, the set $A$ is independent of the choice of $x \in D$.

Theorem 2.9. Let $D$ be a strictly convex bounded domain in a complex Banach space $(X,\|\cdot\|)$ and let $k_{D}$ denote its Kobayashi distance. Let $f: D \rightarrow D$ be $k_{D^{-}}$ nonexpansive, fixed-point-free and condensing with respect to $\alpha_{\|\cdot\|}$ and for each $z \in D$ let $B$ denote the set of all accumulation points, for $s \rightarrow 1^{-}$, of the approximating curve

$$
\left\{h_{f}(s, z)\right\}_{0 \leq s<1}:=\left\{(1-s) z+s f\left(h_{f}(s, z)\right)\right\}_{0 \leq s<1},
$$

i.e.,

$$
B=\left\{x \in \bar{D}: \text { there exists a sequence }\left\{s_{n}\right\} \text { with } 0 \leq s_{n} \rightarrow 1^{-} \text {and } h_{f}\left(s_{n}, z\right) \rightarrow x\right\} .
$$

Then the set $B$ has the following properties:

(i) $B \neq \emptyset$,

(ii) $B \subset \partial D$,

(iii) the set $B$ is independent of the choice of $z \in D$.

Proof. By Lemma 2.6 (ii), if $\left\{s_{n}\right\}$ is a sequence such that $\lim _{n \rightarrow \infty} s_{n}=1,0<$ $s_{n}<1$ and $z \in D$, then the sequence $\left\{x_{n, z}\right\}$, given by $x_{n, z}:=h_{f}\left(s_{n}, z\right)=f_{s_{n}, z}\left(x_{n, z}\right)=$ $\left(1-s_{n}\right) z+s_{n} f\left(x_{n, z}\right)$ for each $n$, contains a norm-convergent subsequence. Since $f$ is fixed-point-free, Theorem 2.7 (vii) implies that for each $z \in D$ we have

$$
\lim _{n \rightarrow \infty} k_{D}\left(x_{n, z}, z\right)=\infty \text {. }
$$

Hence we have $\emptyset \neq B \subset \partial D$. Finally, note that

$$
k_{D}\left(h_{f}(s, z), h_{f}(s, y)\right) \leq k_{D}(z, y)
$$

for $0 \leq s<1$ and each $x, z \in D$ and therefore, by Lemma 2.3, the set $B$ is independent of the choice of $z \in D$.

Directly from Theorems 2.8 and 2.9 we get the following corollary.

Corollary 2.10. Let $D$ be a strictly convex bounded domain in a complex Banach space $(X,\|\cdot\|)$ and let $k_{D}$ denote its Kobayashi distance. Assume that $f: D \rightarrow D$ is $k_{D}$-nonexpansive, fixed-point-free and condensing with respect to $\alpha_{\|\cdot\|}$ and that $C$ is a nonempty, $k_{D}$-closed, convex and $f$-invariant subset of a domain $D$. If the set $A$ is a set of all accumulation points of the sequence $\left\{f^{n}(x)\right\}$, where $x \in D$, and $B$ denote the set of all accumulation points, for $s \rightarrow 1^{-}$and $z \in D$, of the approximating curve

then we have:

$$
\left\{h_{f}(s, z)\right\}_{0 \leq s<1}=\left\{(1-s) z+s f\left(h_{f}(s, z)\right)\right\}_{0 \leq s<1},
$$


(1) $A \subset \bar{C} \cap \partial D$,

(2) $B \subset \bar{C} \cap \partial D$.

Now observe that $k_{D}$-metric segments and compactness of suitable subsets of $D$ are generally used in proofs of the fact that each horosphere is nonempty. Unfortunately, in case of a minimal and $f$-invariant subset $C$ of $D$, which appears in our paper, we are not able to apply the Kobayashi distance $k_{D}$ on $C$, since a nonempty, convex, and $k_{D}$-closed subset from $\tilde{C}$ of $D$ does not have to contain $k_{D}$-metric segments which join points $x, y \in \tilde{C}$. Therefore in our minimal set $C$ we have to introduce the following definition of an intrinsic metric $k_{D, C}$, which is suitable for our aims.

Definition 2.2. Let $D$ be a convex bounded domain in a complex Banach space $(X,\|\cdot\|)$ and let $k_{D}$ denote its Kobayashi distance. Assume that $C$ is a nonempty, $k_{D}$-closed and convex subset of a domain $D$. For $x, y \in C$ an intrinsic metric $k_{D, C}$, which is generated by the Kobayashi distance $k_{D}$, is given by the following formula

$$
\begin{aligned}
k_{D, C}(x, y) & :=\lim _{\eta \rightarrow 0^{+}} \inf \left\{\sum_{j=1}^{n} k_{D}\left(w_{j-1}, w_{j}\right):\left\{w_{0}, w_{1}, \ldots, w_{n}\right\} \in \mathcal{P}_{C, x, y, \eta}\right\}, \\
& =\sup _{\eta>0} \inf \left\{\sum_{j=1}^{n} k_{D}\left(w_{j-1}, w_{j}\right):\left\{w_{0}, w_{1}, \ldots, w_{n}\right\} \in \mathcal{P}_{C, x, y, \eta}\right\},
\end{aligned}
$$

where $\mathcal{P}_{C, x, y, \eta}$ is a set of all finite sequences $\left\{w_{0}, w_{1}, \ldots, w_{n}\right\}$ such that $w_{j} \in C$ for $j=0,1,2, \ldots, n, n \geq 1, w_{0}=x, w_{n}=y$ and $k_{D}\left(w_{j-1}, w_{j}\right) \leq \eta$ for $j=1,2, \ldots, n$.

Finally, we state a definition of a horosphere $G_{D, C}\left(x, \xi, R,\left\{x_{n}\right\}\right)$ in $C \subset D$.

Definition 2.3. Let $D$ be a convex bounded domain in a complex Banach space $(X,\|\cdot\|)$ and let $C$ be a nonempty, $k_{D}$-closed and convex subset of a domain $D$. Assume additionally that $C$ is relatively compact in $(X,\|\cdot\|)$. Let $\left\{x_{n}\right\}$ be a sequence in $C$ with $\lim x_{n}=\xi \in \partial D$ such that all limits

$$
\lim _{n \rightarrow \infty}\left[k_{D, C}\left(w, x_{n}\right)-k_{D, C}\left(z, x_{n}\right)\right]
$$

exist, where $w, z \in C$. A horosphere $G_{D, C}\left(x, \xi, R,\left\{x_{n}\right\}\right)$ in $C$ is introduced in the following way

$$
G_{D, C}\left(x, \xi, R,\left\{x_{n}\right\}\right):=\left\{y \in C: \lim _{n \rightarrow \infty}\left[k_{D, C}\left(y, x_{n}\right)-k_{D, C}\left(x, x_{n}\right)\right]<\frac{1}{2} \log R\right\} .
$$

As we mentioned in Introduction, we give the properties of the intrinsic metric $k_{D, C}$ and the horospheres $G_{D, C}\left(x, \xi, R,\left\{x_{n}\right\}\right)$ in the last section of this paper. We also show the proofs of these properties there.

\section{The Denjoy-Wolff theorem for condensing mappings}

In this section we state and prove our main result on condensing mappings

Theorem 3.1. Let $D$ be a strictly convex bounded domain in an infinite dimensional complex Banach space $(X,\|\cdot\|)$ and let $k_{D}$ denote its Kobayashi distance. If $f: D \rightarrow D$ is $k_{D}$-nonexpansive, fixed-point-free and condensing with respect to $\alpha_{\|\cdot\|}$, then there exists a point $\xi \in \partial D$ such that the sequence $\left\{f^{n}\right\}$ of the iterates of $f$ converges in the compact-open topology to the constant map taking the value $\xi$, i.e. on each compact subset $C$ of $D$, the sequence $\left\{f^{n}\right\}$ tends uniformly to $\xi$. Additionally, an approximating curve $\{x(s, z)\}$ given by the formula

$$
x(s, z):=h_{f}(s, z)=f_{s, z}(x(s, z))=(1-s) z+s f(x(s, z)),
$$


where $0<s<1$ and $z \in D$, is also convergent to $\xi$, when $s \rightarrow 1^{-}$, and a family of approximating curves $\{x(s, \cdot)\}$ tends in the compact-open topology to the constant map taking the value $\xi$, i.e. on each compact subset $C$ of $D$, the family $\{x(s, \cdot)\}$ converges uniformly to $\xi$ for $s \rightarrow 1^{-}$.

Proof. The proof is divided into a few steps.

Step 1. Since $f$ is a $k_{D}$-nonexpansive, fixed-point-free and $\alpha_{\|\cdot\|}$-condensing mapping, by Lemma 2.6 for each $x \in D$ the set of all elements of a sequence of iterates $\left\{f^{n}(x)\right\}$ is relatively compact in $(X,\|\cdot\|)$. Moreover, for each $z \in D$ and for each sequence $\left\{s_{n}\right\}$ such that $\lim _{n \rightarrow \infty} s_{n}=1$ and $0<s_{n}<1$ the set of all elements of the approximating sequence given by

$$
x_{n}:=h_{f}\left(s_{n}, z\right)=f_{s_{n}, z}\left(x_{n}\right)=\left(1-s_{n}\right) z+s_{n} f\left(x_{n}\right)
$$

for $n=1,2,3, \ldots$, is also relatively compact in $(X,\|\cdot\|)$.

Next, let for each $x \in D$ a set $A$ be the set of all accumulation points of the sequence $\left\{f^{n}(x)\right\}$, and for each $z \in D$ let a set $B$ be the set of all accumulation points, for $s \rightarrow 1^{-}$, of the approximating curve

$$
\{x(s, z)\}_{0 \leq s<1}=\left\{h_{f}(s, z)\right\}_{0 \leq s<1}=\left\{(1-s) z+s f\left(h_{f}(s, z)\right)\right\}_{0 \leq s<1} .
$$

Recall that by Theorems 2.8 and 2.9 these sets $A$ and $B$ have the following properties:

(i) $A \neq \emptyset$ and $B \neq \emptyset$,

(ii) the set $A$ is independent of the choice of $x \in D$ and the set $B$ is independent of the choice of $z \in D$,

(iii) $A \subset \partial D$ and $B \subset \partial D$, and if $\tilde{C}$ is a nonempty, convex and $f$-invariant subset of $D$, then $A \cup B \subset \overline{\tilde{C}} \cap \partial D$.

Step 2. Suppose the set $A \cup B$ has two different elements $\xi_{1}$ and $\xi_{2}$. Under this assumption it is easy to observe that each nonempty, convex, $k_{D^{-}}$-closed and $f$-invariant set $\tilde{C} \subset D$ contains the whole open linear segment $\left(\xi_{1}, \xi_{2}\right)$. Hence in $D$ there exists a unique, nonempty, minimal with respect to inclusion, convex, $k_{D^{-}}$closed and $f$-invariant set $C$. It is obvious that $\left(\xi_{1}, \xi_{2}\right) \subset C$ and $A \cup B \subset \bar{C} \cap \partial D$.

Since the set $C$ is nonempty, minimal with respect to inclusion, convex, $k_{D^{-}}$closed, $f$-invariant and unique, we have

$$
\overline{\operatorname{conv}(f(C))} \cap D=C .
$$

Therefore we get

$$
\alpha_{\|\cdot\|}(f(C))=\alpha_{\|\cdot\|}(\overline{\operatorname{conv}(f(C))} \cap D)=\alpha_{\|\cdot\|}(C)
$$

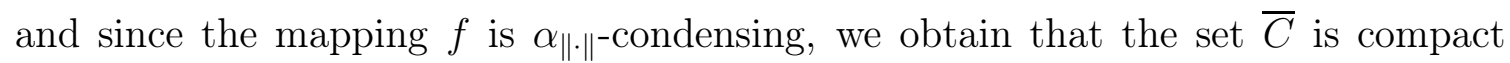
in $(X,\|\cdot\|)$. This means that the metric space $\left(C, k_{D \mid C \times C}\right)$ is complete and finitely compact.

Now we furnish the minimal set $C$ with the intrinsic metric $k_{D, C}$.

Step 3. The set $C$ is $f$-invariant and the mapping $f_{\mid C}$ is $k_{D, C}$-nonexpansive and fixed-point-free. Hence, by the Earle-Hamiltona theorem, norm-compactness of the closure $\bar{C}$ of the set $C$ and by Theorem 2.7, we get the approximating sequence

$$
\left\{x_{n}\right\}=\left\{h_{f}\left(s_{n}, z_{n}\right)\right\}=\left\{f_{s_{n}, z_{n}}\left(x_{n}\right)\right\}=\left\{\left(1-s_{n}\right) z_{n}+s_{n} f\left(x_{n}\right)\right\}
$$

(where $z_{n} \in C, 0<s_{n}<1$ for $n=1,2, \ldots$, and $\lim _{n \rightarrow \infty} s_{n}=1$ ), which is convergent to $\xi \in \partial D$. Now, eventually replacing $\left\{x_{n}\right\}$ by suitably chosen subsequence of this 
sequence, by separability of $C$ we can assume that all limits

$$
\lim _{n \rightarrow \infty}\left[k_{D, C}\left(w, x_{n}\right)-k_{D, C}\left(z, x_{n}\right)\right]
$$

exist for all $w, z \in C$. Next, we fix $x \in C$. Our aim is to show that applying the properties of horospheres $G_{D, C}\left(x, \xi, R,\left\{x_{n}\right\}\right)$ we derive a contradiction with the minimality of the $f$-invariant set $C$.

So, let $y$ be an arbitrary point in $C$. Then we have

$$
\begin{aligned}
x_{n} & =f_{s_{n}, z_{n}}\left(x_{n}\right), \\
\lim _{n \rightarrow \infty} k_{D, C}\left(f(y), f_{s_{n}, z_{n}}(y)\right) & =\lim _{n \rightarrow \infty} k_{D, C}\left(f(y),\left(1-s_{n}\right) z_{n}+s_{n} f(y)\right)=0,
\end{aligned}
$$

and therefore we obtain

$$
\begin{aligned}
& \lim _{n \rightarrow \infty}\left[k_{D, C}\left(f(y), x_{n}\right)-k_{D, C}\left(x, x_{n}\right)\right] \\
& \leq \limsup _{n \rightarrow \infty}\left[k_{D, C}\left(f(y), f_{s_{n}, z_{n}}(y)\right)+k_{D, C}\left(f_{s_{n}, z_{n}}(y), f_{s_{n}, z_{n}}\left(x_{n}\right)\right)-k_{D, C}\left(x, x_{n}\right)\right] \\
& \leq \lim _{n \rightarrow \infty}\left[k_{D, C}\left(y, x_{n}\right)-k_{D, C}\left(x, x_{n}\right)\right] .
\end{aligned}
$$

By the above inequality we get

$$
f\left(C \cap \overline{G_{C, D}\left(x, \xi, R,\left\{x_{n}\right\}\right)}\right) \subset C \cap \overline{G_{D, C}\left(x, \xi, R,\left\{x_{n}\right\}\right)}
$$

for each $R>0$. This means that each set $C \cap \overline{G_{D, C}\left(x, \xi, R,\left\{x_{n}\right\}\right)} \subset C$ is nonempty, convex, $k_{D}$-closed and $f$-invariant. If we take an arbitrary $0<R<1$ then by Theorem 5.9 we see that $C \cap \overline{G_{D, C}\left(x, \xi, R,\left\{x_{n}\right\}\right)}$ is a subset of $C$ which is different from $C$. This contradicts the minimality of $C$. Hence the set $A \cup B$ is a singleton $\{\xi\}$, where $\xi \in \partial D$. This yields to the claimed convergence of the iterating sequences and the approximating curves to $\xi$. Immediately, applying Lemma 2.3, we get the convergence of them in the compact-open topology.

\section{The Denjoy-Wolff theorem for semigroups of holomorphic or $k_{D}$-nonexpansive mappings}

Let $D$ be a bounded and convex domain in a complex Banach space $(X,\|\cdot\|)$. Recall that a family $S=\left\{f_{t}\right\}_{t \geq 0}$ of holomorphic ( $k_{D}$-nonexpansive) self-mappings of $D$ is called a one-parameter continuous semigroup of holomorphic ( $k_{D}$-nonexpansive) mappings if it satisfies the semigroup property:

$$
f_{t} \circ f_{s}=f_{t+s}
$$

for all $s, t \geq 0$ and for each $x \in D$ there exists the strong $\operatorname{limit}_{\lim } \lim _{t \rightarrow 0+} f_{t}(x)=$ $f_{0}(x)=I(x)=x$.

Now we state the following version of the Denjoy-Wolff theorem for semigroups.

Theorem 4.1. Let $D$ be a bounded and strictly convex domain in a complex Banach space $(X,\|\cdot\|)$ and let $S=\left\{f_{t}\right\}_{t \geq 0}$ be a one-parameter continuous semigroup of holomorphic ( $k_{D}$-nonexpansive) mappings in $D$. If $S=\left\{f_{t}\right\}_{t \geq 0}$ contains a fixedpoint-free and condensing with respect to $\alpha_{\|\cdot\|}$ mapping $f_{t_{0}}$, where $t_{0}>0$, then the semigroup $S=\left\{f_{t}\right\}_{t \geq 0}$ has no common fixed point in $D$ and there exists $\xi \in \partial D$ such that $S$ converges to $\bar{\xi}$, as $t$ tends to infinity, uniformly on each compact subset of $D$. 
Proof. The proof is based on the ideas given in [2] and [14]. For the convenience of the reader we present it. Let $C$ be a compact subset of $D$. The semigroup $S=\left\{f_{t}\right\}_{t \geq 0}$ is continuous and each $f_{t}$ is $k_{D}$-nonexpansive. Therefore the set $K=\left\{f_{s}(x): 0 \leq\right.$ $\left.s \leq t_{0}, x \in C\right\} \subset D$ is compact and hence the set $K$ lies strictly inside $D$. This implies that the set $K$ is $k_{D}$-bounded. Next, $f_{t_{0}}$ is fixed-point-free and hence, by Theorem 3.1, there exists $\xi \in \partial D$ such that $f_{n t_{0}}=f_{t_{0}}^{n}$ converges to $\xi$ as $n \rightarrow \infty$, uniformly on each compact subset of $D$. Therefore for the compact sets $C$ and $K$ we have

(a) $f_{t}(x)=f_{n t_{0}+s}(x)=f_{n t_{0}}\left(f_{s}(x)\right) \in f_{n t_{0}}(K)$ for each $x \in C$ and each $t>0$, where $n \in \mathbf{N} \cup 0$ and $0 \leq s<t_{0}$,

(b) $\lim _{n \rightarrow \infty} \operatorname{dist}\left(f_{n t_{0}}(K), \xi\right)=\lim _{n \rightarrow \infty} \sup \left\{\|z-\xi\|: z \in f_{n t_{0}}(K)\right\}=0$.

From these properties we conclude that $\lim _{t \rightarrow \infty} \operatorname{dist}\left(f_{t}(C), \xi\right)=0$, as asserted. Hence we also get that the semigroup $S=\left\{f_{t}\right\}_{t \geq 0}$ has no common fixed point in $D$.

\section{An intrinsic metric in a $k_{D}$-closed and convex subset of a domain $D$}

First, note that the function $k_{D, C}$ satisfies the following inequalities.

Lemma 5.1. Let $D$ be a convex bounded domain in a complex Banach space $(X,\|\cdot\|), k_{D}$ denote its Kobayashi distance, and let $C$ be a nonempty, $k_{D}$-closed and convex subset of a domain $D$. Then for each $\tilde{x} \in C$ and every $R>0$ there exists a constant $M_{\tilde{x}, R}$ such that

$$
\arg \tanh \left(\frac{\|x-y\|}{\operatorname{diam} D}\right) \leq k_{D}(x, y) \leq k_{D, C}(x, y) \leq M_{\tilde{x}, R}\|x-y\|
$$

for each $x, y \in \overline{B_{k_{D}}(\tilde{x}, R)} \cap C$.

Proof. It is obvious that $k_{D}(x, y) \leq k_{D, C}(x, y)$ and by Lemma 2.1 we get

$$
\arg \tanh \left(\frac{\|x-y\|}{\operatorname{diam} D}\right) \leq k_{D}(x, y) \leq k_{D, C}(x, y)
$$

Next, observe that the ball $\overline{B_{k_{D}}(\tilde{x}, R)}$ lies strictly inside $D$ and therefore

$$
r=\operatorname{dist}\left(\overline{B_{k_{D}}(\tilde{x}, R)}, \partial D\right)>0 .
$$

Let $x, y \in \overline{B_{k_{D}}(\tilde{x}, R)} \cap C$. Take $n \in \mathbf{N}$ such that

$$
\frac{\|y-x\|}{n}<\frac{r}{2}
$$

Let $x_{i}=x+\frac{i}{n}(y-x) \in C$ for $i=0,1, \ldots, n$. Then we have $x_{0}=x, x_{n}=y$,

$$
\sup _{0 \leq t \leq \frac{1}{2}} \frac{d \arg \tanh }{d t}(t)=\frac{4}{3}, \quad \operatorname{dist}\left(x_{i}, \partial D\right) \geq r, \quad \frac{\left\|x_{i}-x_{i+1}\right\|}{\operatorname{dist}\left(x_{i}, \partial D\right)}<\frac{1}{2},
$$

and

$$
\arg \tanh \left(\frac{\left\|x_{i}-x_{i+1}\right\|}{\operatorname{dist}\left(x_{i}, \partial D\right)}\right) \leq \frac{4}{3} \frac{\left\|x_{i}-x_{i+1}\right\|}{\operatorname{dist}\left(x_{i}, \partial D\right)} \leq \frac{4}{3 r}\left\|x_{i+1}-x_{i}\right\|=M_{\tilde{x}, R}\left\|x_{i+1}-x_{i}\right\|
$$


for $i=0,1, \ldots, n-1$, where $M_{\tilde{x}, R}=\frac{4}{3 r}$. Hence, for an arbitrary $\eta>0$ and a sufficiently large $n$, we get $k_{D}\left(x_{i}, x_{i+1}\right)<\eta$ for $i=0,1, \ldots, n-1$ and therefore

$$
\sum_{i=0}^{n-1} k_{D}\left(x_{i}, x_{i+1}\right) \leq M_{\tilde{x}, R} \sum_{i=0}^{n-1}\left\|x_{i+1}-x_{i}\right\|=M_{\tilde{x}, R}\|y-x\| .
$$

This implies the claimed inequality

$$
k_{D, C}(x, y) \leq M_{\tilde{x}, R}\|x-y\| .
$$

The above lemma shows that the function $k_{D, C}$ is correctly defined and it is easy to observe that $k_{D, C}$ is really a metric in $C$. This lemma also shows that in the set $C$ the convergence in the norm $\|\cdot\|$ is equivalent to the convergence in the metric $k_{D, C}$, and is also equivalent to the convergence in $k_{D}$. Additionally, the boundedness of a set in $\left(C, k_{D \mid C \times C}\right)$ is equivalent to boundedness of this set in $\left(C, k_{D, C}\right)$. Therefore the metric space $\left(C, k_{D, C}\right)$ is complete. Next, if $\bar{C}$ is compact in $(X,\|\cdot\|)$, then $\left(C, k_{D, C}\right)$ is finitely compact.

We also have the following two simple observations.

Lemma 5.2. Let $D$ be a convex bounded domain in a complex Banach space $(X,\|\cdot\|)$ and let $k_{D}$ denote its Kobayashi distance. Assume that $C$ is a nonempty, $k_{D^{-}}$ closed and convex subset of a domain $D$. Then for each $x, y \in C$ and for each convergent to 0 sequence $\left\{\eta_{i}\right\}_{i}$ of positive numbers there exists sequence $\left\{\left\{w_{0, \eta_{i}}, w_{1, \eta_{i}}, \ldots\right.\right.$, $\left.\left.w_{n_{i}, \eta_{i}}\right\}\right\}_{i}$ such that

(i) $\left\{w_{0, \eta_{i}}, w_{1, \eta_{i}}, \ldots, w_{n_{i}, \eta_{i}}\right\} \in \mathcal{P}_{C, x, y, \eta_{i}}$ for $i=1,2, \ldots$,

(ii) $k_{D, C}(x, y)=\lim _{i} \sum_{j=1}^{n_{i}} k_{D}\left(w_{j-1, \eta_{i}}, w_{j, \eta_{i}}\right)$.

Lemma 5.3. Let $D$ be a convex bounded domain in a complex Banach space $(X,\|\cdot\|)$ and let $k_{D}$ denote its Kobayashi distance. Assume that $C$ is a nonempty, $k_{D}$-closed and convex subset of a domain $D$. If for $x, y \in C$ there exist sequences $\left\{\eta_{i}\right\}_{i}$ and $\left\{\left\{w_{0}, w_{1}, \ldots, w_{n_{i}, \eta_{i}}\right\}\right\}_{i}$, and a constant $L \geq 0$ such that

(a) $\left\{\eta_{i}\right\}_{i}$ is a sequence of positive numbers which converges to 0 ,

(b) $\left\{w_{0, \eta_{i}}, w_{1, \eta_{i}}, \ldots, w_{n_{i}, \eta_{i}}\right\} \in \mathcal{P}_{C, x, y, \eta_{i}}$ for $i=1,2, \ldots$,

(c) $\liminf _{i} \Sigma_{j=1}^{n_{i}} k_{D}\left(w_{j-1, \eta_{i}}, w_{j, \eta_{i}}\right) \leq L$,

then $k_{D, C}(x, y) \leq L$.

Now we recall a definition of a metrically convex metric space.

Definition 5.1. ([58], see also $[10,19])$ Let $(X, d)$ be a metric space. If for all distinct points $x, y \in X$ there exists $z \in X \backslash\{x, y\}$ such that $d(x, z)+d(z, y)=d(x, y)$, then the metric space $(X, d)$ is said to be metrically convex.

We also need a definition of a metric segment.

Definition 5.2. ([58], see also $[10,19])$ Let $(X, d)$ be a metric space and let $x, y$ be two different points in $X$. A subset of $X$, denoted by $[x, y]_{d}$, is called a $d$-metric segment in $X$ joining points $x$ and $y$, if

(i) $x, y \in[x, y]_{d}$;

(ii) for each $0<\beta<d(x, y)$ there exists a unique point $z \in[x, y]_{d}$ such that

$$
d(x, z)=\beta
$$


and

$$
d(x, y)=d(x, z)+d(z, y)
$$

(iii) for each $w \in[x, y]_{d}$ we have

$$
d(x, y)=d(x, w)+d(w, y) .
$$

Here we also have to recall the Menger theorem in the weaker version which is sufficient for our purposes.

Theorem 5.4. ([58], see also $[5,10,11,19,40,59])$ Let $(X, d)$ be a finitely compact metric space. If $(X, d)$ is metrically convex, then any two distinct points in $X$ can be joined by a $d$-metric segment in $X$.

Directly from the definition of the intrinsic metric $k_{D, C}$ and the Menger theorem we get the following lemma.

Lemma 5.5. Let $D$ be a convex bounded domain in a complex Banach space $(X,\|\cdot\|)$ and let $C$ be a nonempty, $k_{D}$-closed, convex and relatively compact in norm subset of a domain $D$. Then for each two different points $w, z \in C$ there exists a $k_{D, C}$-metric segment $[w, z]_{k_{D, C}}$ in $C$, i.e.

(i) the set $[w, z]_{k_{D, C}}$ is a subset of $C$;

(ii) $w, z \in[w, z]_{k_{D, C}}$;

(iii) for each $0<\beta<k_{D, C}(w, z)$ there exists a unique point $v \in[w, z]_{k_{D, C}}$ such that

$$
k_{D, C}(w, v)=\beta
$$

and

$$
k_{D, C}(w, z)=k_{D, C}(w, v)+k_{D, C}(v, z) ;
$$

(iv) for each $v \in[w, z]_{k_{D, C}}$ we have

$$
k_{D, C}(w, z)=k_{D, C}(w, v)+k_{D, C}(v, z) .
$$

Proof. By the Menger theorem it is sufficient to prove that the metric space $\left(C, k_{D, C}\right)$ is metrically convex. Indeed, let $x, y \in C$ be two distinct points. Then by Lemma 5.2 there exist sequences $\left\{\eta_{i}\right\}_{i}$ and $\left\{\left\{w_{0, \eta_{i}}, w_{1, \eta_{i}}, \ldots, w_{n_{i}, \eta_{i}}\right\}\right\}_{i}$ such that

(i) $\left\{\eta_{i}\right\}_{i}$ is a sequence of positive numbers which converges to 0 ,

(ii) $\left\{w_{0, \eta_{i}}, w_{1, \eta_{i}}, \ldots, w_{n_{i}, \eta_{i}}\right\} \in \mathcal{P}_{C, x, y, \eta_{i}}$ for $i=1,2, \ldots$,

(iii) $k_{D, C}(x, y)=\lim _{i} \Sigma_{j=1}^{n_{i}} k_{D}\left(w_{j-1, \eta_{i}}, w_{j, \eta_{i}}\right)$.

Let $\beta=\frac{1}{2} k_{D, C}(x, y)$. Without loss of generality we may assume that $0<\eta_{i}<$ $\frac{1}{4} k_{D}(x, y) \leq \frac{1}{4} k_{D, C}(x, y)$ and $\sum_{j=1}^{n_{i}} k_{D}\left(w_{j-1, \eta_{i}}, w_{j, \eta_{i}}\right)>\frac{1}{2} k_{D, C}(x, y)$ for each $i$. Then for each $i$ we find the smallest $1<j_{i}<n_{i}$ such that

$$
\sum_{j=1}^{j_{i}} k_{D}\left(w_{j-1, \eta_{i}}, w_{j, \eta_{i}}\right) \geq \frac{1}{2} k_{D, C}(x, y)>\sum_{j=1}^{j_{i}-1} k_{D}\left(w_{j-1, \eta_{i}}, w_{j, \eta_{i}}\right) .
$$

Since $\eta_{i} \rightarrow 0^{+}$we get

$$
\lim _{i} \sum_{j=1}^{j_{i}} k_{D}\left(w_{j-1, \eta_{i}}, w_{j, \eta_{i}}\right)=\frac{1}{2} k_{D, C}(x, y)
$$


This and the equality

$$
k_{D, C}(x, y)=\lim _{i} \sum_{j=1}^{n_{i}} k_{D}\left(w_{j-1, \eta_{i}}, w_{j, \eta_{i}}\right)
$$

imply

$$
\lim _{i} \sum_{j=j_{i}+1}^{n_{i}} k_{D}\left(w_{j-1, \eta_{i}}, w_{j, \eta_{i}}\right)=\frac{1}{2} k_{D, C}(x, y) .
$$

By assumption the set $C$ is relatively compact in $(X,\|\cdot\|)$ and $k_{D}$-closed. Therefore the sequence $\left\{w_{j_{i}, \eta_{i}}\right\}_{i}$, which is bounded in $\left(D, k_{D}\right)$, contains a convergent subsequence $\left\{w_{j_{i_{l}}, \eta_{i_{l}}}\right\}_{l}$. Let $v=\lim _{l} w_{j_{i_{l}}, \eta_{i_{l}}} \in C$. Then we have (see Lemma 5.3)

$$
\begin{aligned}
& k_{D, C}(x, v) \leq \lim _{l}\left[\sum_{j=1}^{j_{i_{l}}} k_{D}\left(w_{j-1, \eta_{i}}, w_{j, \eta_{i}}\right)+k_{D}\left(w_{j_{l},}, \eta_{i_{l}}, v\right)\right]=\frac{1}{2} k_{D, C}(x, y), \\
& k_{D, C}(v, y) \leq \lim _{l}\left[k_{D}\left(v, w_{j_{i_{l}}, \eta_{i_{l}}}\right)+\sum_{j=j_{i_{l}+1}}^{n_{i_{l}}} k_{D}\left(w_{j-1, \eta_{i}}, w_{j, \eta_{i}}\right)\right]=\frac{1}{2} k_{D, C}(x, y),
\end{aligned}
$$

and hence we obtain

$$
k_{D, C}(x, y)=k_{D, C}(x, v)+k_{D, C}(v, y) .
$$

This means that $\left(C, k_{D, C}\right)$ is metrically convex.

Before we prove the next properties of $k_{D, C}$, observe the following facts describing behavior of self-mappings in $\left(D, k_{D}\right)$ and their restrictions on $C$ furnished with $k_{D, C}$.

Lemma 5.6. Let $D$ be a convex bounded domain in a complex Banach space $(X,\|\cdot\|)$ and let $C$ be a nonempty, $k_{D}$-closed and convex subset of a domain $D$.

(1) If a mapping $g: D \rightarrow D$ is $k_{D}$-nonexpansive and the set $C$ is $g$-invariant, then the restriction $g_{\mid C}: C \rightarrow C$ of $g$ to $C$ is $k_{D, C}$-nonexpansive.

(2) If a mapping $g: D \rightarrow D$ is a contraction with a constant $0 \leq k<1$ in metric space $\left(D, k_{D}\right)$ and $C$ is $g$-invariant, then $g_{\mid C}: C \rightarrow C$ is a contraction with the same constant $k$ in the metric space $\left(C, k_{D, C}\right)$.

Proof. Let $x, y \in C, \eta>0$ and $\left\{w_{0}, w_{1}, \ldots, w_{n}\right\} \in \mathcal{P}_{C, x, y, \eta}$.

If $g$ is $k_{D}$-nonexpansive, then we have

$$
k_{D}\left(g\left(w_{j-1}\right), g\left(w_{j}\right)\right) \leq k_{D}\left(w_{j-1}, w_{j}\right)
$$

for $j=1,2, \ldots, n$ and therefore $\left\{g\left(w_{0}\right), g\left(w_{1}\right), \ldots, g\left(w_{n}\right)\right\} \in \mathcal{P}_{C, g(x), g(y), \eta}$. Additionally, we get

$$
\sum_{j=1}^{n} k_{D}\left(g\left(w_{j-1}\right), g\left(w_{j}\right)\right) \leq \sum_{j=1}^{n} k_{D}\left(w_{j-1}, w_{j}\right) .
$$

Similarly, if $g$ is $k$-contraction, where $0 \leq k<1$, then we obtain

$$
k_{D}\left(g\left(w_{j-1}\right), g\left(w_{j}\right)\right) \leq k \cdot k_{D}\left(w_{j-1}, w_{j}\right) \leq k_{D}\left(w_{j-1}, w_{j}\right)
$$

for $j=1,2, \ldots, n$ and hence $\left\{g\left(w_{0}\right), g\left(w_{1}\right), \ldots, g\left(w_{n}\right)\right\} \in \mathcal{P}_{C, g(x), g(y), \eta}$. We also have

$$
\sum_{j=1}^{n} k_{D}\left(g\left(w_{j-1}\right), g\left(w_{j}\right)\right) \leq k \cdot \sum_{j=1}^{n} k_{D}\left(w_{j-1}, w_{j}\right) .
$$


Corollary 5.7. Let $D$ be a convex bounded domain in a complex Banach space $(X,\|\cdot\|)$ and let $C$ be a nonempty, $k_{D}$-closed and convex subset of a domain $D$. Then

(a) if $x, y, z \in C$ and $s \in[0,1]$, then

$$
k_{D, C}(s x+(1-s) y, s x+(1-s) z) \leq k_{D, C}(y, z) ;
$$

(b) if $x, y \in C$ and $s, t \in[0,1]$, then

$$
k_{D, C}(s x+(1-s) y, t x+(1-t) y) \leq k_{D, C}(x, y) .
$$

Proof. (a) Let $x, y, z \in C$ and $0<s<1$. It is sufficient to apply the contraction $g: D \rightarrow D$ given by $g=(1-s) x+I$ (see Theorem 2.4 and Lemma 5.6) to get

$$
k_{D, C}(s x+(1-s) y, s x+(1-s) z) \leq k_{D, C}(y, z) .
$$

(b) Observe that for $0 \leq s^{\prime} \leq 1$ and $w, z \in C$ we get

$$
k_{D, C}\left(s^{\prime} w+\left(1-s^{\prime}\right) z, z\right)=k_{D, C}\left(s^{\prime} w+\left(1-s^{\prime}\right) z, s^{\prime} z+\left(1-s^{\prime}\right) z\right) \leq k_{D, C}(w, z)
$$

by (a), and

$$
s x+(1-s) y=\frac{s-t}{1-t} x+\left(1-\frac{s-t}{1-t}\right)[t x+(1-t) y]
$$

for $0 \leq t \leq s<1$ and $x, y \in C$.

We can generalize the property (a) from the last corollary in the following way (compare with Lemma 2.2).

Theorem 5.8. Let $D$ be a convex bounded domain in a complex Banach space $(X,\|\cdot\|)$ and let $C$ be a nonempty, $k_{D}$-closed and convex subset of a domain $D$. Then for $x, y, w, z \in C$ and $s \in[0,1]$ we have

$$
k_{D, C}(s x+(1-s) y, s w+(1-s) z) \leq \max \left[k_{D, C}(x, w), k_{D, C}(y, z)\right] .
$$

Proof. Consider the following cases.

a) Let $s \in(0,1), x, y, w, z \in C$ and $y=z$. Then we can apply Corollary 5.7 (a).

b) Let $x, y, w, z \in C, x \neq w, y \neq z$ and $s \in(0,1)$. Without loss of generality we may assume that $0<k_{D, C}(y, z) \leq k_{D, C}(x, w)$. Fix $n_{0} \in \mathbf{N}$ such that $0<\frac{1}{n_{0}}<$ $\frac{1}{4} k_{D}(y, z)$. Then for each $n_{0} \leq n \in \mathbf{N}$ there exist $0<\delta_{n}<\frac{1}{3 n}, m_{1, n} \in \mathbf{N}$ and $\left\{u_{0, n}, u_{1, n}, \ldots, u_{m_{1, n}, n}\right\} \in \mathcal{P}_{C, x, w, \delta_{n}}$ satisfying the inequality

$$
k_{D, C}(x, w)-\frac{1}{n}<\sum_{j=1}^{m_{1, n}} k_{D}\left(u_{j-1, n}, u_{j, n}\right)<k_{D, C}(x, w)+\frac{1}{n},
$$

and $u_{i, n} \neq u_{j, n}$ for $i \neq j$ and $i, j=1,2, \ldots, m_{1, n}$. Then

$$
\mu_{n}=\min _{j, j^{\prime}=0,1, \ldots m_{1, n}, j \neq j^{\prime}} k_{D}\left(u_{j, n}, u_{j^{\prime}, n}\right)>0
$$

and let $\rho_{n}=\frac{\mu_{n}}{5 m_{1, n}}<\frac{\delta_{n}}{4 m_{1, n}}$.

Next, there exist $0<\eta_{n}<\rho_{n}, m_{2, n} \in \mathbf{N}$ and $\left\{v_{0, n}, v_{1, n}, \ldots, v_{m_{2, n}, n}\right\} \in \mathcal{P}_{C, y, z, \eta_{n}}$ such that $v_{i, n} \neq v_{i^{\prime}, n}$ for $i \neq i^{\prime}, i, i^{\prime}=0,1,2, \ldots, m_{2, n}$ and

$$
k_{D, C}(y, z)-\rho_{n}<\sum_{i=0}^{m_{2, n}-1} k_{D}\left(v_{i, n}, v_{i+1, n}\right)<k_{D, C}(y, z)+\rho_{n} .
$$


Now we choose $r_{0, n}, r_{1, n}, \ldots, r_{m_{n}^{\prime}, n}$ from indexes $0,1, \ldots, m_{2, n}$ in such way that

$$
\begin{array}{r}
0=r_{0, n}<\cdots<r_{m_{n}^{\prime}, n} \leq m_{2, n}, \quad 1 \leq m_{n}^{\prime} \leq m_{1, n} \\
\sum_{i=r_{0, n}}^{r_{1, n}-2} k_{D}\left(v_{i, n}, v_{i+1, n}\right)<k_{D}\left(u_{0, n}, u_{1, n}\right) \leq \sum_{i=r_{0, n}}^{r_{1, n}-1} k_{D}\left(v_{i, n}, v_{i+1, n}\right), \\
\sum_{i=r_{1, n}}^{r_{2, n}-2} k_{D}\left(v_{i, n}, v_{i+1, n}\right)<k_{D}\left(u_{1, n}, u_{2, n}\right) \leq \sum_{i=r_{1, n}}^{r_{2, n}-1} k_{D}\left(v_{i, n}, v_{i+1, n}\right)
\end{array}
$$

and we continue this procedure as far as it is possible, i.e. we finish our procedure when

$$
\sum_{i=r_{m_{n}^{\prime}-1, n}}^{r_{m_{n}^{\prime}, n}-2} k_{D}\left(v_{i, n}, v_{i+1, n}\right)<k_{D}\left(u_{m_{n}^{\prime}-1, n}, u_{m_{n}^{\prime}, n}\right) \leq \sum_{i=r_{m_{n}^{\prime}-1, n}}^{r_{m_{n}^{\prime}, n}-1} k_{D}\left(v_{i, n}, v_{i+1, n}\right)
$$

and either $r_{m_{n}^{\prime}, n}=m_{2, n}$ or $r_{m_{n}^{\prime}, n}<m_{2, n}$. Then we have the following possible cases.

Case 1. $m_{n}^{\prime}<m_{1, n}$. Then we set $\tilde{u}_{j, n}=u_{j, n}$ for $j=0,1, \ldots, m_{1, n}, \tilde{u}_{m_{1, n}+1, n}=w$, $\tilde{v}_{j, n}=v_{r_{j, n}, n}$ for $j=1, \ldots, m_{n}^{\prime}$ and $\tilde{v}_{j, n}=z$ for $j=m_{n}^{\prime}+1, \ldots, m_{1, n}+1$.

Observe that

$$
k_{D}\left(\tilde{u}_{j-1, n}, \tilde{u}_{j, n}\right)=k_{D}\left(u_{j-1, n}, u_{j, n}\right)<\delta_{n},
$$

for $j=1, \ldots, m_{1, n}$,

$$
k_{D}\left(\tilde{u}_{m_{1, n}, n}, \tilde{u}_{m_{1, n}+1, n}\right)=k_{D}(w, w)=0
$$

and

$$
\begin{aligned}
k_{D}\left(\tilde{v}_{j-1, n}, \tilde{v}_{j, n}\right) & =k_{D}\left(v_{r_{j-1, n}, n}, v_{r_{j, n}, n}\right) \leq \sum_{i=r_{j-1, n}}^{r_{j, n}-1} k_{D}\left(v_{i, n}, v_{i+1, n}\right) \\
& =\sum_{i=r_{j-1, n}}^{r_{j, n}-2} k_{D}\left(v_{i, n}, v_{i+1, n}\right)+k_{D}\left(v_{r_{j, n}-1, n}, v_{r_{j, n}, n}\right) \\
& <k_{D}\left(u_{j-1, n}, u_{j, n}\right)+\eta_{n}<2 \delta_{n}
\end{aligned}
$$

for $j=1, \ldots, m_{n}^{\prime}$.

Now we have to consider two possibilities: either $r_{m_{n}^{\prime}, n}=m_{2, n}$ or $r_{m_{n}^{\prime}, n}<m_{2, n}$.

If $r_{m_{n}^{\prime}, n}=m_{2, n}$, then we get

$$
k_{D}\left(\tilde{v}_{j-1, n}, \tilde{v}_{j, n}\right)=k_{D}(z, z)=0
$$

for $j=m_{n}^{\prime}+1, \ldots, m_{1, n}+1$.

If $r_{m_{n}^{\prime}, n}<m_{2, n}$, then we have

$$
\begin{aligned}
k_{D}\left(\tilde{v}_{m_{n}^{\prime}, n}, \tilde{v}_{m_{n}^{\prime}+1, n}\right) & =k_{D}\left(v_{r_{m_{n}^{\prime}, n}}, z\right)=k_{D}\left(v_{r_{m_{n}^{\prime}, n}}, v_{m_{2, n}, n}\right) \\
& \leq \sum_{i=r_{m_{n}^{\prime}, n}}^{m_{2, n}-1} k_{D}\left(v_{i, n}, v_{i+1, n}\right)<k_{D}\left(u_{m_{n}^{\prime}}, u_{m_{n}^{\prime}+1}\right)<\delta_{n}
\end{aligned}
$$

and

$$
k_{D}\left(\tilde{v}_{j-1, n}, \tilde{v}_{j, n}\right)=k_{D}(z, z)=0
$$

for $j=m_{n}^{\prime}+2, \ldots, m_{1, n}+1$. 
Hence, in both subcases, we obtain

$$
\left\{\tilde{u}_{0, n}, \ldots, \tilde{u}_{m_{1, n}+1, n}\right\} \in \mathcal{P}_{C, x, w, 2 \delta_{n}}
$$

and

which implies

$$
\left\{\tilde{v}_{0, n}, \ldots, \tilde{v}_{m_{1, n}+1, n}\right\} \in \mathcal{P}_{C, y, z, 2 \delta_{n}}
$$

$$
\begin{aligned}
\left\{s \tilde{u}_{0, n}+(1-s) \tilde{v}_{0, n}, \ldots, s \tilde{u}_{m_{1, n}+1, n}+(1-s) \tilde{v}_{m_{1, n}+1, n}\right\} & \in \mathcal{P}_{C, s x+(1-s) y, s w+(1-s) z, 2 \delta_{n}} \\
& \subset \mathcal{P}_{C, s x+(1-s) y, s w+(1-s) z, \frac{2}{n}} .
\end{aligned}
$$

Additionally, we get

$$
\begin{aligned}
& k_{D}\left(s \tilde{u}_{j-1, n}+(1-s) \tilde{v}_{j-1, n}, s \tilde{u}_{j, n}+(1-s) \tilde{v}_{j, n}\right) \\
& \leq \max \left[k_{D}\left(\tilde{u}_{j-1, n}, \tilde{u}_{j, n}\right), k_{D}\left(\tilde{v}_{j-1, n}, \tilde{v}_{j, n}\right)\right]=\max \left[k_{D}\left(u_{j-1, n}, u_{j, n}\right), k_{D}\left(\tilde{v}_{j-1, n}, \tilde{v}_{j, n}\right)\right] \\
& \leq \max \left[k_{D}\left(u_{j-1, n}, u_{j, \eta}\right), k_{D}\left(u_{j-1, n}, u_{j, n}\right)+\eta_{n}\right]=k_{D}\left(u_{j-1, n}, u_{j, n}\right)+\eta_{n}
\end{aligned}
$$

for $j=1, \ldots, m_{n}^{\prime}$,

$$
\begin{aligned}
& k_{D}\left(s \tilde{u}_{j-1, n}+(1-s) \tilde{v}_{j-1, n}, s \tilde{u}_{j, n}+(1-s) \tilde{v}_{j, n}\right) \\
& =k_{D}\left(s u_{j-1, n}+(1-s) z, s u_{j, n}+(1-s) z\right) \\
& \leq k_{D}\left(u_{j-1, n}, u_{j, n}\right)<k_{D}\left(u_{j-1, n}, u_{j, n}\right)+\eta_{n}
\end{aligned}
$$

for $j=m_{n}^{\prime}+1, \ldots, m_{1, n}$, and

$$
\begin{aligned}
& k_{D}\left(s \tilde{u}_{m_{1, n}, n}+(1-s) \tilde{v}_{m_{1, n}, n}, s \tilde{u}_{m_{1, n}+1, n}+(1-s) \tilde{v}_{m_{1, n}+1, n}\right) \\
& =k_{D}(s w+(1-s) z, s w+(1-s) z)=0 .
\end{aligned}
$$

Therefore, we have

$$
\begin{aligned}
& \sum_{j=1}^{m_{1, n}+1} k_{D}\left(s \tilde{u}_{j-1, n}+(1-s) \tilde{v}_{j-1, n}, s \tilde{u}_{j, n}+(1-s) \tilde{v}_{j, n}\right) \\
= & \sum_{j=1}^{m_{1, n}} k_{D}\left(s \tilde{u}_{j-1, n}+(1-s) \tilde{v}_{j-1, n}, s \tilde{u}_{j, n}+(1-s) \tilde{v}_{j, n}\right) \\
< & \sum_{j=1}^{m_{1, n}}\left[k_{D}\left(u_{j-1, n}, u_{j, n}\right)+\eta_{n}\right]=\sum_{j=1}^{m_{1, n}} k_{D}\left(u_{j-1, n}, u_{j, n}\right)+m_{1, n} \eta_{n} \\
< & k_{D, C}(x, w)+\frac{1}{n}+\delta_{n}<\max \left\{k_{D, C}(x, w), k_{D, C}(y, z)\right\}+\frac{2}{n} .
\end{aligned}
$$

Case 2. $m_{n}^{\prime}=m_{1, n}$. Then we set $\tilde{u}_{j, n}=u_{j, n}$ and $\tilde{v}_{j, n}=v_{r_{j, n}, n}$ for $j=1, \ldots, m_{n}^{\prime}=$ $m_{1, n}$. Similarly as in Case 1 we get

$$
\begin{aligned}
& k_{D}\left(\tilde{v}_{j-1, n}, \tilde{v}_{j, n}\right)=k_{D}\left(v_{r_{j-1, n}, n}, v_{r_{j, n}, n}\right) \\
& \leq \sum_{i=r_{j-1, n}}^{r_{j, n}-1} k_{D}\left(v_{i, n}, v_{i+1, n}\right)=\sum_{i=r_{j-1, n}}^{r_{j, n}-2} k_{D}\left(v_{i, n}, v_{i+1, n}\right)+k_{D}\left(v_{r_{j, n}-1, n}, v_{r_{j, n}, n}\right) \\
& <k_{D}\left(u_{j-1, n}, u_{j, n}\right)+\eta_{n}<2 \delta_{n}
\end{aligned}
$$

for $j=1, \ldots, m_{n}^{\prime}=m_{1, n}$.

Next, we have to consider two subcases: either $r_{m_{n}^{\prime}, n}=m_{2, n}$ or $r_{m_{n}^{\prime}, n}<m_{2, n}$. 
If $r_{m_{n}^{\prime}, n}=m_{2, n}$, then we set $\tilde{u}_{m_{1, n}+1, n}=u_{m_{1, n}, n}=w$ and $\tilde{v}_{m_{1, n}+1, n}=v_{r_{m_{n}^{\prime}, n}}=$ $v_{m_{2, n}, n}=z$. Hence we have

$$
k_{D}\left(\tilde{u}_{m_{1, n}, n}, \tilde{u}_{m_{1, n}+1, n}\right)=k_{D}(w, w)=0
$$

and

$$
k_{D}\left(\tilde{v}_{m_{1, n}, n}, \tilde{v}_{m_{1, n}+1, n}\right)=k_{D}(z, z)=0 .
$$

If $r_{m_{n}^{\prime}, n}<m_{2, n}$, then we put $\tilde{u}_{m_{1, n}+1, n}=u_{m_{1, n}, n}=w$ and $\tilde{v}_{m_{1, n}+1, n}=v_{m_{2, n}, n}=z$. Hence we obtain $k_{D}\left(\tilde{u}_{m_{1, n}, n}, \tilde{u}_{m_{1, n}+1, n}\right)=k_{D}(w, w)=0$ and

$$
\begin{aligned}
& k_{D}\left(\tilde{v}_{m_{1, n}, n}, \tilde{v}_{m_{1, n}+1, n}\right)=k_{D}\left(v_{r_{m_{n}^{\prime}, n}, n}, z\right)=k_{D}\left(v_{r_{m_{n}, n}, n}, v_{m_{2, n}, n}\right) \\
& \leq \sum_{i=r_{m_{n}^{\prime}, n}}^{m_{2, n}-1} k_{D}\left(v_{i, n}, v_{i+1, n}\right)=\sum_{i=0}^{m_{2, n}-1} k_{D}\left(v_{i, n}, v_{i+1, n}\right)-\sum_{i=0}^{r_{m_{n}^{\prime}, n}-1} k_{D}\left(v_{i, n}, v_{i+1, n}\right) \\
& \leq k_{D, C}(y, z)+\rho_{n}-\sum_{j=0}^{m_{n}^{\prime}-1}\left[\sum_{i=r_{j, n}}^{r_{j+1, n}-1} k_{D}\left(v_{i, n}, v_{i+1, n}\right)\right] \\
& \leq k_{D, C}(y, z)+\rho_{n}-\sum_{j=0}^{m_{1, n}-1} k_{D}\left(u_{j, \eta}, u_{j+1, \eta}\right)<k_{D, C}(y, z)+\rho_{n}-\left[k_{D, C}(x, w)-\frac{1}{n}\right] \\
& <k_{D, C}(x, w)+\frac{2}{n}-k_{D, C}(x, w)=\frac{2}{n} .
\end{aligned}
$$

Observe that, in both subcases, we get

$$
\left\{\tilde{u}_{0, n}, \ldots, \tilde{u}_{m_{1, n}+1, n}\right\} \in \mathcal{P}_{C, x, w, \frac{2}{n}}
$$

and

Thus

$$
\left\{\tilde{v}_{0, n}, \ldots, \tilde{v}_{m_{1, n}+1, n}\right\} \in \mathcal{P}_{C, y, z, \frac{2}{n}}
$$

$$
\left\{s \tilde{u}_{0, n}+(1-s) \tilde{v}_{0, n}, \ldots, s \tilde{u}_{m_{1, n}+1, n}+(1-s) \tilde{v}_{m_{1, n}+1, n}\right\} \in \mathcal{P}_{C, s x+(1-s) y, s w+(1-s) z, \frac{2}{n}} .
$$

We also have

$$
\begin{aligned}
& k_{D}\left(s \tilde{u}_{j-1, n}+(1-s) \tilde{v}_{j-1, n}, s \tilde{u}_{j, n}+(1-s) \tilde{v}_{j, n}\right) \\
& \leq \max \left[k_{D}\left(\tilde{u}_{j-1, n}, \tilde{u}_{j, n}\right), k_{D}\left(\tilde{v}_{j-1, n}, \tilde{v}_{j, n}\right)\right]=\max \left[k_{D}\left(u_{j-1, n}, u_{j, n}\right), k_{D}\left(\tilde{v}_{j-1, n}, \tilde{v}_{j, n}\right)\right] \\
& \leq \max \left[k_{D}\left(u_{j-1, n}, u_{j, n}\right), k_{D}\left(u_{j-1, n}, u_{j, n}\right)+\eta_{n}\right] \\
& =k_{D}\left(u_{j-1, \eta}, u_{j, \eta}\right)+\eta_{n}<\delta_{n}+\eta_{n}<\frac{2}{n}
\end{aligned}
$$

for $j=1, \ldots, m_{n}^{\prime}=m_{1, n}$ and

$$
\begin{aligned}
& k_{D}\left(s \tilde{u}_{m_{1, n}, n}+(1-s) \tilde{v}_{m_{1, n}, n}, s \tilde{u}_{m_{1, n}+1, n}+(1-s) \tilde{v}_{m_{1, n}+1, n}\right) \\
& \leq \max \left\{k_{D}\left(\tilde{u}_{m_{1, n}, n}, \tilde{u}_{m_{1, n}+1, n}\right), k_{D}\left(\tilde{v}_{m_{1, n}, n}, \tilde{v}_{m_{1, n}+1, n}\right)\right\}<\frac{2}{n} .
\end{aligned}
$$

So similarly as in Case 1 we get

$$
\begin{aligned}
& \sum_{j=1}^{m_{1, n}+1} k_{D}\left(s \tilde{u}_{j-1, n}+(1-s) \tilde{v}_{j-1, n}, s \tilde{u}_{j, n}+(1-s) \tilde{v}_{j, n}\right) \\
& <\max \left\{k_{D, C}(x, w), k_{D, C}(y, z)\right\}+\frac{4}{n} .
\end{aligned}
$$


Summarizing, in the both cases, we obtain

$$
\begin{aligned}
\left\{w_{0, n}, \ldots, w_{m_{1, n}+1, n}\right\} & =\left\{s \tilde{u}_{0, n}+(1-s) \tilde{v}_{0, n}, \ldots, s \tilde{u}_{m_{1, n}+1, n}+(1-s) \tilde{v}_{m_{1, n}+1, n}\right\} \\
& \in \mathcal{P}_{C, s x+(1-s) y, s w+(1-s) z, \frac{2}{n}} \\
k_{D}\left(w_{j-1, n}, w_{j, n}\right) & =k_{D}\left(s \tilde{u}_{j-1, n}+(1-s) \tilde{v}_{j-1, n}, s \tilde{u}_{j, n}+(1-s) \tilde{v}_{j, n}\right) \\
& \leq k_{D}\left(u_{j-1, \eta}, u_{j, \eta}\right)+\eta_{n}
\end{aligned}
$$

for $j=1, \ldots, m_{1, n}$ and

$$
\begin{aligned}
& k_{D}\left(w_{m_{1, n}, n}, w_{m_{1, n}+1, n}\right) \\
& =k_{D}\left(s \tilde{u}_{m_{1, n}, n}+(1-s) \tilde{v}_{m_{1, n}, n}, s \tilde{u}_{m_{1, n}+1, n}+(1-s) \tilde{v}_{m_{1, n}+1, n}\right)<\frac{2}{n} .
\end{aligned}
$$

Hence we get

$$
\begin{aligned}
& \sum_{j=0}^{m_{j, n}+1} k_{D}\left(w_{j-1, n}, w_{j, n}\right)=\sum_{j=0}^{m_{1, n}} k_{D}\left(w_{j-1, n}, w_{j, n}\right)+k_{D}\left(w_{m_{1, n}, n}, w_{m_{1, n}+1, n}\right) \\
& <\sum_{j=1}^{m_{1, n}}\left[k_{D}\left(u_{j-1, n}, u_{j, n}\right)+\eta_{n}\right]+\frac{2}{n}=\sum_{j=1}^{m_{1, n}} k_{D}\left(u_{j-1, n}, u_{j, n}\right)+m_{1, n} \cdot \eta_{n}+\frac{2}{n} \\
& <k_{D, C}(x, w)+\frac{4}{n}=\max \left\{k_{D, C}(x, w), k_{D, C}(y, z)\right\}+\frac{4}{n}
\end{aligned}
$$

which implies

$$
\begin{aligned}
k_{D, C}(s x+(1-s) y, s w+(1-s)) & \leq \liminf _{n} \Sigma_{j=0}^{m_{j, n}+1} k_{D}\left(w_{j-1, n}, w_{j, n}\right) \\
& \leq \max \left\{k_{D, C}(x, w), k_{D, C}(y, z)\right\}
\end{aligned}
$$

and completes the proof.

Finally, we investigate a horosphere $G_{D, C}\left(x, \xi, R,\left\{x_{n}\right\}\right)$ in a nonempty, $k_{D^{-}}$closed, convex and relatively compact subset $C$ of a bounded and convex domain $D$ in a complex Banach space $(X,\|\cdot\|)$ (see Definition 2.3). Taking under account local $k_{D, C}$-compactness of the set $C$ and known properties of the metric $k_{D, C}$, we are able to prove the following properties of this horosphere.

Theorem 5.9. Under the assumptions given in Definition 2.3 the horosphere $G_{D, C}\left(x, \xi, R,\left\{x_{n}\right\}\right) \subset C$ has the following properties:

(I) for each $x \in C$ and for each $R>0$ the horosphere $G_{D, C}\left(x, \xi, R,\left\{x_{n}\right\}\right)$ is nonempty and convex;

(II) for each $0<R<1$ we have $B_{k_{D, C}}\left(x,-\frac{1}{2} \log R\right) \cap G_{D, C}\left(x, \xi, R,\left\{x_{n}\right\}\right)=\emptyset$ and therefore $\overline{G_{D, C}\left(x, \xi, R,\left\{x_{n}\right\}\right)} \cap C \neq C$.

Proof. (I) By Lemma 5.5 for each $w, z \in C, w \neq z, \beta \in \mathbf{R}$ and $0<\beta<$ $k_{D, C}(w, z)$, there exists a point $y_{w, z, \beta}$ in $C$ such that

$$
k_{D, C}(w, z)=k_{D, C}\left(w, y_{w, z, \beta}\right)+k_{D, C}\left(y_{w, z, \beta}, z\right)
$$

and

$$
k_{D, C}\left(y_{w, z, \beta}, z\right)=\beta .
$$

Fix $0<\beta \in \mathbf{R}$ and take $\beta>\max \left\{0,-\frac{1}{2} \log R\right\}$. Without loss of generality we can assume that $0<\beta<k_{D, C}\left(x_{n}, x\right)$ for each $n \in \mathbf{N}$. Then for each $y_{x_{n}, x, \beta}$ we have

$$
k_{D, C}\left(x_{n}, x\right)=k_{D, C}\left(x_{n}, y_{x_{n}, x, \beta}\right)+k_{D, C}\left(y_{x_{n}, x, \beta}, x\right)=k_{D}\left(x_{n}, y_{x_{n}, x, \beta}\right)+\beta \text {. }
$$


Since the sequence $\left\{y_{x_{n}, x, \beta}\right\}_{n}$ lies strictly inside $D$, it has a norm convergent subsequence $\left\{y_{x_{n_{i}}, x, \beta}\right\}_{i}$ with a limit $y_{\beta, x} \in C$. Hence we get

$$
\beta=\lim _{i \rightarrow \infty} k_{D, C}\left(y_{x_{i}, x, \beta}, x\right)=k_{D, C}\left(y_{\beta, x}, x\right)
$$

and

$$
\begin{aligned}
& \lim _{n \rightarrow \infty}\left[k_{D, C}\left(y_{\beta, x}, x_{n}\right)-k_{D, C}\left(x, x_{n}\right)\right] \\
& =\lim _{i \rightarrow \infty}\left[k_{D, C}\left(y_{x_{n_{i}}, x, \beta}, x_{n_{i}}\right)-k_{D, C}\left(x, x_{n_{i}}\right)\right] \\
& =-\lim _{i \rightarrow \infty} k_{D, C}\left(y_{x_{n_{i}}, x, \beta}, x\right)=-\beta<\frac{1}{2} \log R .
\end{aligned}
$$

Therefore we obtain $y_{\beta, x} \in G_{D, C}\left(x, \xi, R,\left\{x_{n}\right\}\right)$. Next, applying Theorem 5.8 we immediately get the convexity of $G_{D, C}\left(x, \xi, R,\left\{x_{n}\right\}\right)$.

(II) Fix $0<R<1$ and $y \in B_{k_{D, C}}\left(x,-\frac{1}{2} \log R\right)$. Then we have

$$
\lim _{n \rightarrow \infty}\left[k_{D, C}\left(y, x_{n}\right)-k_{D, C}\left(x, x_{n}\right)\right] \geq-k_{D, C}(y, x)>\frac{1}{2} \log R
$$

and therefore $y \notin G_{D, C}\left(x, \xi, R,\left\{x_{n}\right\}\right)$.

Acknowledgments. The author was partially supported by the Polish MNiSW grant N N201 393737. These results have been partially achieved within the framework of the STREVCOMS Project No 612669 with funding from the IRSES Scheme FP7 Programme of the European Union.

\section{References}

[1] Abate, M.: Horospheres and iterates of holomorphic maps. - Math. Z. 198, 1988, 225-238.

[2] AbAte, M.: Iteration theory of holomorphic maps on taut manifolds. - Mediterranean Press, 1989 .

[3] Abate, M., and J. Raissy: Wolff-Denjoy theorems in non-smooth convex domains. - Preprint.

[4] Akhmerov, R. R., M. I. Kamenskit, A. S. Potapov, A. E. Rodkina, and B. N. Sadovskit: Measures of noncompactness and condensing operators. - Birkhäuser, 1992.

[5] Aronsztajn, T.: Neuer Beweis der Streckenverbundenheit völlstandinger konvexer Räume. Ergebnisse eines mathematischen Kolloquiums (Wien) 6, 1935, 45-56.

[6] Ayerbe Toledano, J. M., T. Domínguez Benavides, and G. López Acedo: Measures of noncompactness in metric fixed point theory. - Birkhäuser, 1997.

[7] Banaś, J., and K. Goebel: Measures of noncompactness in Banach spaces. - Marcel Dekker, 1980 .

[8] Beardon, A. F.: Iteration of contractions and analytic maps. - J. London Math. Soc. 41, 1990, 141-150.

[9] Beardon, A. F.: The dynamics of contractions. - Ergodic Theory Dynam. Systems 17, 1997, 1257-1266.

[10] Blumenthal, L. M.: Theory and applications of distance geometry. - The Clarendon Press, Oxford, 1953.

[11] Blumenthal, L. M., and K. Menger: Studies in geometry. - W. H. Freeman Co., San Francisco, 1970.

[12] Budzyńska, M.: The Denjoy-Wolff theorem in $\mathbf{C}^{n}$. - Nonlinear Anal. 75, 2012, 22-29.

[13] Budzyńska, M., and T. Kuczumow: Theorems of Denjoy-Wolff type for a family of $k_{D^{-}}$ nonexpansive retracts. - J. Nonlinear Conv. Anal. (to appear). 
[14] Budzyńska, M., T. Kuczumow, and S. Reich: Theorems of Denjoy-Wolff type. - Ann. Mat. Pura Appl. 192, 2013, 621-648.

[15] Budzyńska, M., T. Kuczumow, and S. Reich: The Denjoy-Wolff theorem for compact holomorphic mappings in reflexive Banach spaces. - J. Math. Anal. Appl. 396, 2012, 504-512.

[16] Budzyńska, M., T. Kuczumow, and S. Reich: The Denjoy-Wolff theorem for compact holomorphic mappings in complex Banach spaces. - Ann. Acad. Sci. Fenn. Math. 38, 2013, $747-756$.

[17] Burckel, R. B.: An introduction to classical complex analysis, Volume I. - Birkhäuser, 1979.

[18] Burckel, R. B.: Iterating analytic self-maps of discs. - Amer. Math. Monthly 88, 1981, 396407.

[19] Busemann, H.: The geometry of geodesics. - Academic Press, New York, 1955.

[20] Catka, A.: On conditions under which isometries have bounded orbits. - Colloq. Math. 48, $1984,219-227$.

[21] Chen, G. N.: Iteration for holomorphic maps of the open unit ball and the generalized upper half-plane of $\mathbf{C}^{n}$. - J. Math. Anal. Appl. 98, 1984, 305-313.

[22] Chu, C.-H., and P. Mellon: Iteration of compact holomorphic maps on a Hilbert ball. Proc. Amer. Math. Soc. 125, 1997, 1771-1777.

[23] Denjoy, A.: Sur l'itération des fonctions analytiques. - C. R. Acad. Sci. Paris 182, 1926, $255-257$.

[24] Dineen, S.: The Schwarz lemma. - Clarendon Press, 1989.

[25] Dineen, S., and R. M. Timoney: Complex geodesics on convex domains. - In: Progress in Functional Analysis (Peñíscola, 1990), North-Holland Math. Stud. 170, North-Holland, Amsterdam, 1992, 333-365.

[26] Dineen, S., R. M. Timoney, and J.-P. Vigué: Pseudodistances invariantes sur les domaines d'un espace localement convexe. - Ann. Sc. Norm. Super. Pisa 12, 1985, 515-529.

[27] Earle, C. J., and R. S. Hamilton: A fixed point theorem for holomorphic mappings. - Proc. Symp. Pure Math. 16, 1970, 61-65.

[28] Edelstein, M.: On non-expansive mappings of Banach spaces. - Proc. Cambridge Phil. Soc. $60,1964,439-447$.

[29] Engelking, R.: Outline of general topology. - Elsevier, 1968.

[30] Franzoni, T., and E. Vesentini: Holomorphic maps and invariant distances. - NorthHolland, 1980.

[31] Goebel, K., and W. A. Kirk: Topics in metric fixed point theory. - Cambridge Univ. Press, 1990.

[32] Goebel, K., and S. Reich: Iterating holomorphic self-mappings of the Hilbert ball. - Proc. Japan Acad. 58, 1982, 349-352.

[33] Goebel, K., and S. Reich: Uniform convexity, hyperbolic geometry, and nonexpansive mappings. - Marcel Dekker, 1984.

[34] HaRris, L. A.: Schwarz-Pick systems of pseudometrics for domains in normed linear spaces. - In: Advances in Holomorphy, North Holland, 1979, 345-406.

[35] Hervé, M.: Quelques propriétés des applications analytiques d'une boule à m dimensions dans elle-même. - J. Math. Pures Appl. 42, 1963, 117-147.

[36] Jarnicki, M., and P. Pflug: Invariant distances and metrics in complex analysis. - Walter de Gruyter, 1993.

[37] Kapeluszny, J., T. Kuczumow, and S. Reich: The Denjoy-Wolff theorem in the open unit ball of a strictly convex Banach space. - Adv. Math. 143, 1999, 111-123. 
[38] Kapeluszny, J., T. Kuczumow, and S. Reich: The Denjoy-Wolff theorem for condensing holomorphic mappings. - J. Funct. Anal. 167, 1999, 79-93.

[39] Karlson, A.: Non-expanding maps and Busemann functions. - Ergodic Theory Dynam. Systems 21, 2001, 1447-1457.

[40] Kink, W. A.: Caristi's fixed point theorem and metric convexity. - Colloq. Math. 36, 1976, $81-86$.

[41] Kobayashi, S.: Invariant distances on complex manifolds and holomorphic mappings. - J. Math. Soc. Japan 19, 1967, 460-480.

[42] Kobayashi, S.: Hyperbolic manifolds and holomorphic mappings. - Marcel Dekker, 1970.

[43] Kobayashi, S.: Hyperbolic complex spaces. - Springer, 1998.

[44] Kryczka, A., and T. Kuczumow: The Denjoy-Wolff-type theorem for compact $k_{B_{H^{-}}}$ nonexpansive maps on a Hilbert ball. - Ann. Univ. Mariae Curie-Skłodowska Sect. A 51, 1997, $179-184$.

[45] Kubota, Y.: Iteration of holomorphic maps of the unit ball into itself. - Proc. Amer. Math. Soc. $88,1983,476-480$.

[46] Kuczumow, T., S. Reich, and D. Shoikhet: Fixed points of holomorphic mappings: a metric approach. - In: Handbook of Metric Fixed Point Theory (W. A. Kirk and B. Sims, eds.), Kluwer Academic Publishers, 2001, 437-515.

[47] Kuczumow, T., and A. Stachura: Iterates of holomorphic and $k_{D}$-nonexpansive mappings in convex domains in $\mathbf{C}^{n}$. - Adv. Math. 81, 1990, 90-98.

[48] Kuczumow, T., and A. Stachura: The Denjoy-Wolff theorem for $s$-condensing mappings. - Ann. Univ. Mariae Curie-Skłodowska Sect. A 53, 1999, 109-115.

[49] Kuratowski, K.: Sur les espaces complets. - Fund. Math. 15, 1930, 301-309.

[50] Kuratowski, K.: Topology, Volume I. - Academic Press \& PWN, 1966.

[51] Lempert, L.: La métrique de Kobayashi et la représentation des domaines sur la boule. - Bull. Soc. Math. France 109, 1981, 427-474.

[52] LEMPERT, L.: Holomorphic retracts and intrinsic metrics in convex domains. - Anal. Math. 8, $1982,257-261$.

[53] Lins, B.: A Denjoy-Wolff theorem for Hilbert metric nonexpansive maps on polyhedral domains. - Math. Proc. Cambridge Philos. Soc. 143, 2007, 157-164.

[54] Lins, B., and R. Nussbaum: Iterated linear maps on a cone and Denjoy-Wolff theorems. Linear Algebra Appl. 416, 2006, 615-626.

[55] Lins, B., and R. Nussbaum: Denjoy-Wolff theorems, Hilbert metric nonexpansive maps and reproduction-decimation operators. - J. Funct. Anal. 254, 2008, 2365-2386.

[56] MacCluer, B. D.: Iterates of holomorphic self-maps of the open unit ball in $\mathbf{C}^{n}$. - Michigan Math. J. 30, 1983, 97-106.

[57] Mellon, P.: A generall Wolff theorem for arbitrary Banach spaces. - Math. Proc. R. Ir. Acad. 104A, 2004, 127-142.

[58] Menger, K.: Untersuchungen über allgemeine Metrik I, II, III. - Math. Ann. 100, 1928, $75-163$.

[59] Menger, K., and A. N. Milgram: On linear sets in metric spaces. - Reports of a Mathematical Colloquium, 2nd series, Issue 1, 1939, 16-17.

[60] Mercer, P.: Complex geodesics and iterates of holomorphic maps on convex domains in $\mathbf{C}^{n}$. - Trans. Amer. Math. Soc. 338, 1993, 201-211.

[61] Nussbaum, R.: Fixed point theorems and Denjoy-Wolff theorems for Hilbert's projective metric in infinite dimensions. - Topol. Methods Nonlinear Anal. 29, 2007, 199-249. 
[62] Poggi-Corradini, P.: On the failure of a generalized Denjoy-Wolff theorem. - Conform. Geom. Dyn. 6, 2002, 13-32.

[63] Reich, S.: Averaged mappings in the Hilbert ball. - J. Math. Anal. Appl. 109, 1985, 199-206.

[64] ReIch, S.: Fixed point theory in the Hilbert ball. - Contemp. Math. 72, 1988, 225-232.

[65] Reich, S., and D. Shoikhet: The Denjoy-Wolff theorem. - Ann. Univ. Mariae CurieSkłodowska Sect. A 51:2, 1997, 219-240.

[66] Reich, S., and D. Shoikhet: Nonlinear semigroups, fixed points and geometry of domains in Banach spaces. - Imperial College Press, London, 2005.

[67] Sadovskit, B. N.: A fixed point principle. - Funct. Anal. Appl. 1, 1967, 151-153.

[68] Sine, R.: Behavior of iterates in the Poincaré metric. - Houston J. Math. 15, 1989, 273-289.

[69] Stachura, A.: Iterates of holomorphic self-maps of the unit ball in Hilbert spaces. - Proc. Amer. Math. Soc. 93, 1985, 88-90.

[70] Vesentini, E.: Su un teorema di Wolff e Denjoy. - Rend. Sem. Mat. Fis. Milano 53, 1983, $17-25$.

[71] Wolff, J.: Sur l'itération des fonctions holomorphes dans une région, et dont les valeurs appartiennent à cette région. - C. R. Acad. Sci. Paris 182, 1926, 42-43.

[72] Wolff, J.: Sur l'itération des fonctions bornées. - C. R. Acad. Sci. Paris 182, 1926, 200-201.

[73] Wolff, J.: Sur une généralisation d'un théorème de Schwarz. - C. R. Acad. Sci. Paris 182, $1926,918-920$.

Received 4 February $2014 \bullet$ Accepted 11 April 2014 